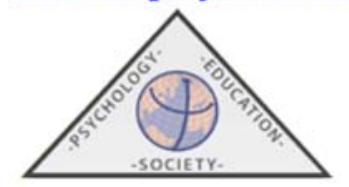

\title{
Revisión de las formas abreviadas de la Escala de Inteligencia de Wechsler para Adultos
}

\author{
Raquel ÚBEDA*, Inmaculada FUENTES****y Carmen DASÍ* \\ *Universidad de Valencia, **CIBERSAM, España
}

(Received on February 1, 2015; Accepted on February 21, 2015)

RESUMEN: El presente trabajo tiene como objetivo presentar una revisión teórica de las principales formas abreviadas que han sido desarrolladas por varios autores a partir de las distintas versiones de la Escala de inteligencia de Wechsler para adultos que han ido surgiendo a lo largo del tiempo. El desarrollo de formas cortas ha ido creciendo en paralelo a la aparición de las nuevas versiones de la escala completa. Una forma abreviada permite estimar la capacidad intelectual con un tiempo de administración menor, por lo que puede ser de gran utilidad si el objetivo de evaluación es obtener una medida general de la capacidad intelectual.

Palabras clave: WAIS, WAIS-R, WAIS-III, WAIS-IV, estimación CI.

Wechsler Adult Intelligence Scale: Review of short forms

\begin{abstract}
The aim of this study is to present a theoretical review of the main short forms that have been developed by several authors based on different versions of the Wechsler Adult Intelligence Scale. The development of short forms has been growing parallel to the appearance of new versions of the full scale. A short form allows estimating the intellectual capacity of someone with less time of administration, which could be very useful if the assessment objective is to obtain an overall measure of intellectual capacity.
\end{abstract}

Keywords: WAIS, WAIS-R, WAIS-III, WAIS-IV, IQ estimation.

Correspondencia: Inmaculada Fuentes Durá. Facultad de Psicología. Universidad de Valencia. Avda. Blasco Ibañez, 21, 46010 Valencia. Spain. Email: inmaculada.fuentes@uv.es.

\section{Introducción}

La Escala de inteligencia de Weschler para adultos es el test más frecuentemente utilizado en la evaluación de la inteligencia en una amplia variedad de escenarios o contextos, incluyendo la práctica clínica (Blyler, Gold, Iannone y Buchanan, 2000; Girard, Axelrod y Wilkins, 2010; Kaufman y Lichtenberger, 2006). Desde su primer desarrollo hasta la actualidad se han elaborado diferentes versiones. La primera versión fue realizada por David Wechsler en el año 1939 (Escala de inteligencia de Wechsler-Bellevue; Wechsler, 1939). Esta escala constaba de 11 pruebas (ver tabla 1), y de las siguientes puntuaciones: verbal, manipulativa y 
total. Más tarde, en el año 1955, Wechsler desarrolló la primera versión de la Escala de inteligencia de Wechsler para adultos (WAIS) (Wechsler, 1955), en la incluyó las mismas pruebas que la Wechsler-Bellevue pero con algunos cambios en cuanto a los ítems, criterios de aplicación y puntuación. Las pruebas se clasificaban en dos escalas, la verbal y la manipulativa: CI verbal (CIV), CI manipulativo (CIM) y CI total (CIT). En el año 1981, se publicó la Wechsler Adult Intelligence Scale-Revised (WAIS-R), que mantuvo las mismas pruebas y puntuaciones que su predecesora (ver tabla 1 ).

Tabla 1. Principales Características de las Versiones de la Escala de Inteligencia de Wechsler para Adultos (Wechsler-Bellevue, WAIS y WAIS-R)

\begin{tabular}{|c|c|c|c|c|c|c|}
\hline \multirow{3}{*}{ Edad de aplicación } & \multirow{2}{*}{\multicolumn{2}{|c|}{ Wechsler-Bellevue (1939) }} & \multirow{2}{*}{\multicolumn{2}{|c|}{ WAIS (1955) }} & \multirow{2}{*}{\multicolumn{2}{|c|}{ WAIS-R (1981) }} \\
\hline & & & & & & \\
\hline & \multicolumn{2}{|c|}{ 7-69 años } & \multicolumn{2}{|c|}{ 16-64 años } & \multicolumn{2}{|c|}{ 16-74 años } \\
\hline \multirow[t]{7}{*}{ Estructura } & Escala verbal: & Escala manipulativa: & Escala verbal: & Escala manipulativa: & Escala verbal: & Escala manipulativa: \\
\hline & Información & Clave de números & Información & Clave de números & Información & Clave de números \\
\hline & Comprensión & Figuras incompletas & Comprensión & Figuras incompletas & Comprensión & Figuras incompletas \\
\hline & Aritmética & Cubos & Aritmética & Cubos & Aritmética & Cubos \\
\hline & Semejanzas & Historietas & Semejanzas & Historietas & Semejanzas & Historietas \\
\hline & Dígitos & Rompecabezas & Dígitos & Rompecabezas & Dígitos & Rompecabezas \\
\hline & \multicolumn{2}{|l|}{ Vocabulario } & \multicolumn{2}{|l|}{ Vocabulario } & \multicolumn{2}{|l|}{ Vocabulario } \\
\hline Puntuaciones obtenidas & \multicolumn{2}{|l|}{ CIV, CIM, CIT } & \multicolumn{2}{|l|}{ CIV, CIM, CIT } & \multicolumn{2}{|l|}{ CIV, CIM, CIT } \\
\hline
\end{tabular}

Nota. Abreviaturas: CIV = cociente intelectual verbal; CIM = cociente intelectual manipulativo; CIT = cociente intelectual total

En 1997, apareció la Escala de inteligencia de Wechsler para adultos-III (WAIS-III) (Wechsler, 1997). Esta escala mantuvo las 11 pruebas de la WAIS-R y añadió tres nuevas (ver tabla 2). Se mantuvieron las tres puntuaciones tradicionales: CIV, CIM y CIT y se incorporaron cuatro nuevas puntuaciones: comprensión verbal (ICV), organización perceptiva (IOP), memoria de trabajo (IMT) y velocidad de procesamiento (IVP). La Escala de inteligencia de Wechsler para adultos-IV (WAIS-IV) (Wechsler, 2008) es la última versión, conserva 12 pruebas de la WAIS-III e incorpora tres nuevas (ver tabla 2). Permite obtener puntuaciones en cuatro índices: Índice de comprensión verbal (ICV), Índice de razonamiento perceptivo (IRP), Índice de memoria de trabajo, (IMT) e Índice de velocidad de procesamiento (IVP) y también ofrece una puntuación total (CIT). En esta versión ya no se contempla el CIV y CIM.

La duración promedio de administración de las pruebas principales de la WAIS-IV, según su Manual, es de alrededor de 67 minutos para la población general (Wechsler, 2008), incrementándose dicha duración en grupos clínicos. El intento de disminuir el tiempo de administración es una de las razones de la elaboración de formas abreviadas de este instrumento. El desarrollo de formas breves se remonta prácticamente a la aparición de la primera versión y ha ido en paralelo a la aparición de las sucesivas versiones. Las formas abreviadas permiten realizar una estimación del CI con un tiempo de administración menor, que puede llegar a reducirse del 25 al 50\% respecto al tiempo total (Ryan, López y Werth, (c) Psy, Soc, \& Educ, 2016, Vol. 8(1) 
1998; Ward, Selby y Clark, 1987). Además, sus buenos índices de fiabilidad y validez las hacen superiores a los instrumentos breves de medida de CI disponibles actualmente (Kaufman, 1990; Reynolds, Willson y Clark, 1983).

Tabla 2. Principales Características de las Versiones de la Escala de Inteligencia de Wechsler para Adultos-III y IV (WAIS-III y WAIS-IV)

\begin{tabular}{|c|c|c|c|c|c|c|c|c|}
\hline & \multicolumn{4}{|c|}{ WAIS-III (1997) } & \multicolumn{4}{|c|}{ WAIS-IV (2008) } \\
\hline Edad de & \multicolumn{4}{|c|}{ 16-89 años } & \multicolumn{4}{|c|}{ 16-89 años y 11 meses } \\
\hline \multirow[t]{5}{*}{ Estructura } & Escala & erbal: & \multicolumn{2}{|c|}{ Escala manipulativa: } & $\begin{array}{l}\text { Índice de } \\
\text { Comprensión } \\
\text { verbal: }\end{array}$ & $\begin{array}{l}\text { Índice de } \\
\text { Memoria de } \\
\text { trabajo: }\end{array}$ & $\begin{array}{l}\text { Índice de } \\
\text { Razonamiento } \\
\text { perceptivo: }\end{array}$ & $\begin{array}{l}\text { Índice de } \\
\text { Velocidad de } \\
\text { procesamiento: }\end{array}$ \\
\hline & CV: & MT: & OP: & VP: & Vocabulario & Aritmética & $\begin{array}{l}\text { Figuras } \\
\text { incompletas }\end{array}$ & Clave de números \\
\hline & Vocabulario & & $\begin{array}{l}\text { Figuras } \\
\text { incompletas }\end{array}$ & $\begin{array}{l}\text { Clave de } \\
\text { números }\end{array}$ & Semejanzas & Dígitos & Cubos & $\begin{array}{l}\text { Búsqueda de } \\
\text { símbolos }\end{array}$ \\
\hline & $\begin{array}{l}\text { Semejanzas } \\
\text { Información }\end{array}$ & $\begin{array}{l}\text { Dígitos } \\
\text { Letras y } \\
\text { números* }\end{array}$ & $\begin{array}{l}\text { Cubos } \\
\text { Matrices* }\end{array}$ & $\begin{array}{l}\text { Búsqueda } \\
\text { de } \\
\text { símbolos* }\end{array}$ & $\begin{array}{l}\text { Información } \\
\text { Comprensión }\end{array}$ & $\begin{array}{l}\text { Letras y } \\
\text { números }\end{array}$ & $\begin{array}{l}\text { Matrices } \\
\text { Puzles visuales* }\end{array}$ & Cancelación* \\
\hline & & & & & & & Balanzas* & \\
\hline
\end{tabular}

Puntuaciones CIV, CIM, CIT

obtenidas

Nota. Abreviaturas: CV = Comprensión verbal; MT = Memoria de trabajo; OP = Organización perceptiva; VP = Velocidad de procesamiento; ICV = Índice de Comprensión verbal; IRP = Índice de Razonamiento perceptivo; IMT = Índice de Memoria de trabajo; IVP = Índice de Velocidad de procesamiento; CIT = cociente intelectual total

* Prueba de creación nueva

Su uso se justifica cuando no se requiere realizar una evaluación exhaustiva de la capacidad intelectual, sino que el objetivo es obtener una rápida estimación de la inteligencia (Kaufman, 1990; Crawford, Allum y Kinion, 2008). También resultan útiles en la evaluación de grupos clínicos en los que hay dificultades atencionales y/o motivacionales, o en aquellos en los que la fatiga puede aparecer pronto (Caplan, 1983; Paolo y Ryan, 1991).

El objetivo del presente trabajo es realizar una revisión de las principales formas abreviadas que han sido desarrolladas a partir de las distintas versiones de la Escala de inteligencia de Wechsler para adultos.

\section{Método}

Los trabajos sobre los que se ha realizado la presente revisión fueron identificados a través de las siguientes bases de datos: Dialnet, PsycINFO y Medline. El proceso de búsqueda se realizó para los artículos publicados hasta Mayo de 2015 sin restricción de lenguaje. En la primera búsqueda se incluyeron los términos "formas abreviadas" ó "formas cortas" y "Escala de inteligencia de Wechsler para adultos" en el título del documento. Una segunda búsqueda se centro en aspectos más específicos. Se incluyeron los términos: "formas abreviadas" ó "formas cortas" y "Wechsler-Bellevue"; "formas abreviadas" ó ”formas cortas” y "WAIS"; "formas 
abreviadas” ó "formas cortas” y "WAIS-R"; “formas abreviadas” ó ”formas cortas” y "WAISIII"; "formas abreviadas" ó "formas cortas" y "WAIS-IV". Las búsquedas electrónicas proporcionaron 120 artículos y dos más fueron encontrados en las referencias. Sólo se han incluido en esta revisión los 30 artículos en los que desarrollaba una forma breve de alguna de las versiones de la Escala de inteligencia de Wechsler para adultos.

\section{Resultados}

Las primeras investigaciones que propusieron una escala breve como alternativa para la evaluación de la capacidad intelectual de una persona en un corto período de tiempo parten de los cuestionarios Binet-Simon (Doll, 1917) y Stanford-Binet (Terman y Merrill, 1937).Tras la publicación de la Escala de inteligencia de Wechsler-Bellevue (Wechsler, 1939), Rabin (1943) llevó a cabo el primer estudio de una forma corta de dicha escala, en la que incluyó tres pruebas de la Escala verbal (ver tabla 3). Para estimar los CIs de la escala original prorrateó las puntuaciones (multiplicándolas por 10/3) y consultó las tablas elaboradas por Wechsler para la obtención de los CIs. Dichos CIs obtuvieron una elevada correlación con los encontrados en la escala completa.

Con la aparición de la primera versión de la Escala de inteligencia de Wechsler para adultos (WAIS; Wechsler, 1955), aumentó el número de estudios sobre formas abreviadas. Doppelt (1956), fue el primero que propuso una forma abreviada de esta versión, estando compuesta por dos tests verbales y dos manipulativos (ver Tabla 3). Dicha forma abreviada proporcionaba una estimación válida de la puntuación completa (correlaciones entre 0.92 y 0.97) tanto para muestras psiquiátricas como para la muestra de estandarización (Guertin, Rabin, Frank y Ladd, 1961). Maxwell, en 1957, realizó un estudio, con una muestra de 300 personas, en el que determinó la correlación de todas las posibles combinaciones de dos, tres, cuatro y cinco tests con la forma completa de la WAIS, estableciendo así una mayor validez de criterio. Concluyó que la mejor forma abreviada es la que combina tests verbales y manipulativos, y que la exactitud de las formas abreviadas depende del número de tests incluidos en ellas (la correlación aumenta a medida que se incrementa el número de tests).

Wolfson y Bachelis (1960), propusieron una nueva metodología de reducción de la escala completa basada en la reducción del número de ítems administrados en cada subtest y en la reducción del número de los propios subtests. La forma abreviada resultante consiste en una selección de cada tercer ítem de Información y Vocabulario; los ítems impares de Comprensión, Aritmética y Semejanzas; y los ítems de Dígitos completos. Las puntuaciones directas de cada prueba han de multiplicarse por un determinado factor (dos o tres dependiendo del procedimiento de reducción empleado en cada test). A continuación, han de convertirse en puntuaciones típicas y, sumando estas últimas, se obtiene el CI siguiendo el procedimiento usual.

Una forma abreviada que alcanzó gran popularidad es la desarrollada por Satz y Mogel (1962). Estos autores proponen realizar la misma selección de ítems para los tests verbales que la propuesta por Wolfson y Bachelis (1960) e introducen que, para los tests manipulativos, se selecciona cada tercer ítem de Figuras incompletas, y los ítems impares del resto de subtests manipulativos, exceptuando Clave de números que se aplica en su totalidad. Se obtiene una 
estimación del CI verbal, CI manipulativo y CI total. En el estudio obtuvieron correlaciones con la escala completa que oscilaban entre 0.97 y 0.99 .

Britton y Savage (1966) propusieron una forma breve formada por cuatro subtests (ver tabla 3) a partir de los cuales es posible obtener las puntuaciones típicas total, verbal (sumando las puntuaciones de Comprensión y Vocabulario y multiplicando el resultado por tres) y manipulativa (multiplicando por 5/2 la suma de Cubos y Rompecabezas). La correlación obtenida con el CIT tras aplicar esta forma en una muestra de ancianos fue de 0.97.

A partir de la recopilación de las selecciones de las mejores combinaciones de tests de la WAIS, Silverstein (1970) y Zimmerman y Woo-Sam (1973) coincidieron en afirmar que la mejor pareja es la formada por Vocabulario y Cubos, y el mejor trío el formado por Información, Vocabulario y Cubos. En España, el estudio de formas abreviadas de la WAIS es limitado, existiendo únicamente el trabajo de Robles, Ramón y Cabrera (1990) en el que aplicaron la reducción de la escala elaborada por Britton y Savage (1966) a una muestra de pacientes psiquiátricos. Concluyeron que tanto la escala completa como la forma abreviada pueden ser usadas indistintamente para la obtención del CI de este grupo de pacientes.

Tabla 3. Formas Abreviadas de las Versiones de la Escala de Inteligencia de Wechsler para Adultos: Wechsler-Bellevue, WAIS y WAIS-R

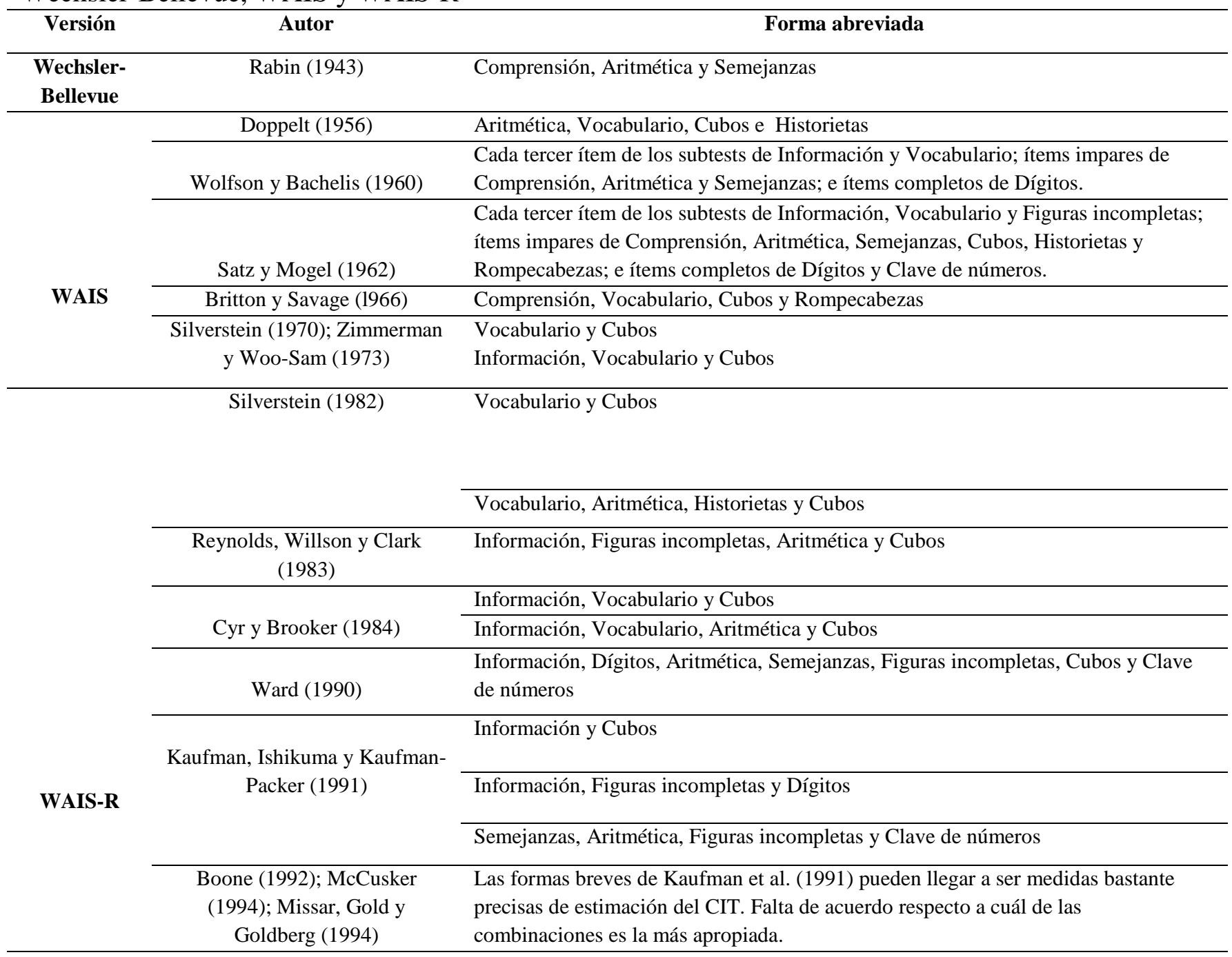


La forma abreviada de Ward (1990) es la que permite obtener estimaciones más

Allen et al. (1998)

precisas del CI, seguida por la forma abreviada de cuatro subtests de Kaufman et al. (1991).

Benedict, Schretlen y Bobholtz

(1992); Ryan, Weilage y $\quad$ La forma abreviada de Ward (1990) estima con mayor precisión el CI.

Spaulding (1999)

Con la aparición de la revisión de la Escala de inteligencia de Wechsler para adultos (WAIS-R) (Wechsler, 1981), se incrementó el estudio de las formas breves. Se seleccionaron formas breves a partir de los datos de la muestra de estandarización para posteriormente pasar a demostrar su utilidad con muestras especiales. Ya no se intentaba descubrir la mejor forma abreviada, sino validarlas para demostrar mejores propiedades psicométricas.

Silverstein (1982) desarrolló dos formas abreviadas de la WAIS-R: una compuesta por dos subtests y la otra por cuatro subtests (ver Tabla 3). La primera propuesta coincide con la forma abreviada de la WAIS-R desarrollada a partir de una muestra de personas diagnosticadas con esquizofrenia realizada por Sumiyoshi, Uetsuki, Suga, Kasai y Sumiyoshi (2013). La segunda propuesta coincide con la efectuada por Doppelt (1956), el cual obtuvo una validez de 0.91 y una fiabilidad de 0.94 .

Cyr y Brooker (1984) con los datos de la muestra de estandarización de la WAIS-R, calcularon los coeficientes de validez y fiabilidad para todas las combinaciones posibles de dos, tres, cuatro y cinco tests y seleccionaron: Información, Vocabulario y Cubos como el mejor trío y, como el mejor cuarteto, añadieron el test de Aritmética. Por otro lado, Ward (1990) desarrolló una forma abreviada formada por siete subtests (ver Tabla 3). Permite realizar una estimación del CI verbal, CI manipulativo y CI total (correlaciones con la forma completa de $0.97,0.96$ y 0.98 respectivamente).

Kaufman, Ishikuma y Kaufman-Packer (1991) propusieron tres formas abreviadas (ver Tabla 3) compuestas respectivamente por una pareja de subtests, un trío (validez de 0.91 y una fiabilidad de 0.92) y un cuarteto formado por una pareja de pruebas verbales y otra manipulativa (tabla 3) que correlacionaba sustancialmente con sus respectivos CIs y con la que obtuvieron unos índices de validez de 0.93 y de fiabilidad de 0.95 . Las investigaciones que compararon las formas cortas de la WAIS-R expuestas, en muestras de pacientes psiquiátricos, concluyeron que las formas abreviadas de Kaufman et al. (1991) pueden llegar a ser medidas bastante precisas del CI total, aunque no mostraron acuerdo respecto a cuál de las combinaciones es la más apropiada. Para Boone (1992), la forma corta compuesta por tres subtests era la mejor opción para estimar el CI total. McCusker (1994), obtuvo que la forma reducida compuesta por el cuarteto muestra el coeficiente de validez más alto (0.93), seguida por la de la triada (0.92) y la de la pareja (0.87). Para Missar, Gold y Goldberg (1994) la mejor forma de estimación del CI total era el cuarteto $(r=.93)$.

Otros autores obtuvieron, a partir de sus estudios con muestras de pacientes psiquiátricos, que la forma abreviada propuesta por Ward (1990) es la que estima con mayor precisión el CI total con una reducción considerable del tiempo de administración y una baja tasa de clasificación errónea según categorías de funcionamiento intelectual (Allen et al., 1998; Benedict, Schretlen y Bobholtz, 1992; Ryan, Weilage y Spaulding, 1999). Allen et al. (1998) 
matizaron que, si se desea realizar una evaluación todavía más rápida, la mejor opción es la forma abreviada de cuatro subtests de Kaufman et al. (1991).

Con el surgimiento de la Escala de inteligencia de Wechsler para adultos-III (WAISIII)(Wechsler, 1997), Pilgrim, Meyers, Bayless y Whetstone (1999) analizaron la forma reducida propuesta por Ward (1990). Obtuvieron correlaciones con la escala completa del WAIS-III, para el CI verbal, CI manipulativo y CI total, casi idénticas a las obtenidas por este autor (0.97, 0.95 y 0.98 respectivamente). Los valores de Axelrod, Ryan y Ward (2001), también con la propuesta de Ward (1990), son para el CI total y CI verbal de 0.97, y para el CI manipulativo de 0.94 .

Tabla 4. Formas Abreviadas de la Escala de Inteligencia de Wechsler para Adultos-III y IV (WAIS-III y WAIS-IV)

\begin{tabular}{|c|c|c|}
\hline Versión & Autor & Forma abreviada \\
\hline \multirow{6}{*}{$\begin{array}{l}\text { WAIS- } \\
\text { III }\end{array}$} & $\begin{array}{l}\text { Pilgrim, Meyers, Bayless y } \\
\text { Whetstone (1999) }\end{array}$ & $\begin{array}{l}\text { Información, Dígitos, Aritmética, Semejanzas, Figuras incompletas, Cubos y Clave } \\
\text { de números (aplicación de la propuesta de Ward (1990) al WAIS-III). }\end{array}$ \\
\hline & $\begin{array}{l}\text { Blyler, Gold, Iannone y Buchanan } \\
\text { (2000) }\end{array}$ & Información, Cubos, Aritmética y Búsqueda de símbolos \\
\hline & Axelrod, Ryan y Ward (2001) & $\begin{array}{l}\text { Figuras incompletas, Clave de números, Semejanzas, Aritmética, Dígitos, } \\
\text { Información y Matrices. }\end{array}$ \\
\hline & Christensen, Girard y Bagby (2007) & $\begin{array}{l}\text { Vocabulario, Semejanzas, Aritmética, Dígitos, Figuras incompletas, Matrices, Clave } \\
\text { de números y Búsqueda de símbolos. }\end{array}$ \\
\hline & Crawford, Allum y Kinion (2008) & Vocabulario, Semejanzas, Cubos, Matrices, Aritmética, Dígitos y Clave de números \\
\hline & $\begin{array}{l}\text { Fuentes-Durá, Romero-Peris, Dasí- } \\
\text { Vivó y Ruiz-Ruiz (2010) }\end{array}$ & $\begin{array}{l}\text { Semejanzas, Clave de números, Figuras incompletas y Dígitos (forma abreviada en } \\
\text { español) }\end{array}$ \\
\hline \multirow{3}{*}{$\begin{array}{l}\text { WAIS- } \\
\text { IV }\end{array}$} & $\begin{array}{l}\text { Meyers, Zellinger, Kockler, Wagner } \\
\text { y Mellado-Miller (2013) }\end{array}$ & $\begin{array}{l}\text { Información, Dígitos, Aritmética, Semejanzas, Figuras incompletas, Cubos y Clave } \\
\text { de números (aplicación de la propuesta de Ward (1990) al WAIS-IV). }\end{array}$ \\
\hline & $\begin{array}{c}\text { Girard, Axelrod, Patel y Crawford } \\
\text { (2014) }\end{array}$ & Información y Clave de números \\
\hline & Denney, Ringe y Lacritz (2015) & Vocabulario y Cubos \\
\hline
\end{tabular}

Con el WAIS-III se desarrollaron también algunas formas cortas para su uso con población psiquiátrica. La propuesta por Christensen, Girard y Bagby (2007) está compuesta por 8 subtest $\left(R^{2}=.97\right)$ (tabla 4$)$. La publicada por Blyler et al. (2000) es aplicable a pacientes diagnosticados de esquizofrenia y se compone de un subtest perteneciente a cada uno de los cuatro factores de la escala $\left(R^{2}=.92\right)$ (tabla 4). Los autores recomiendan su utilidad en el ámbito clínico y de investigación, para personas pertenecientes a dicha población. Siguiendo a estos autores, Fuentes-Durá, Romero-Peris, Dasí-Vivó y Ruiz-Ruiz (2010) desarrollaron una forma corta para su uso con pacientes diagnosticados de esquizofrenia a partir de población española (ver Tabla 4). La forma corta que propusieron obtuvo una correlación significativa y positiva $(r=.91, p<.001)$ con la escala completa, no existiendo diferencias estadísticamente significativas entre los CIs de ambas formas. Además, esta forma breve era capaz de clasificar a un número considerable de personas en la misma categoría de CI que la escala completa (>70\%).

Crawford et al. (2008) desarrollaron una forma breve compuesta por siete subtests (ver Tabla 4) basándose para su elección en la fiabilidad y saturaciones factoriales de los subtests y en la opinión de expertos. 
En el estudio llevado a cabo por Girard et al. (2010) en el que se compararon las formas abreviadas desarrolladas por Axelrod et al. (2001), Christensen et al. (2007), y Crawford et al. (2008), se obtuvo que las tres formas resultan adecuadas en la estimación de los CI verbal, CI manipulativo y CI total; aunque la forma corta propuesta por Axelrod et al. (2001) es la que proporciona la mejor combinación global en términos de propiedades psicométricas y disminución del tiempo de administración. No obstante, la propuesta de Christensen et al. (2007) representa mejor los cuatro factores de la escala y permite obtener, además, medidas de la velocidad de procesamiento.

\section{WAIS-IV}

Sobre la última versión de la prueba, Escala de inteligencia de Wechsler para adultos (WAIS-IV) (Wechsler, 2008), dada su recencia existen pocos estudios. Meyers, Zellinger, Kockler, Wagner y Mellado-Miller (2013) examinaron la utilidad de la forma abreviada propuesta por Ward (1990) en una muestra clínica. Aunque la última versión ha suprimido el CIV y CIM y, en su lugar, incluye los índices de Comprensión verbal (ICV), Razonamiento perceptivo (IRP), Memoria de trabajo (IMT) y Velocidad de procesamiento (IVP), es posible estimarlos. Las correlaciones entre los índices (ICV, IRP, IMT, IVP) y el CIT estimados con respecto a la escala completa fueron altas: $0.97,0.95,1.0,0.92$ y 0.99 respectivamente. Por lo que concluyeron que es posible utilizar la forma breve de siete subtests propuesta por Ward (1990) como una forma corta de la WAIS-IV.

Para Girard, Axelrod, Patel y Crawford (2014) los subtests de Información y Clave de números es la pareja que presenta mayor fiabilidad $\left(r_{x x}=.92\right)$ y validez $\left(r^{\prime}=.85\right)$ en la estimación del CIT. Denney, Ringe y Lacritz (2015) concluyen que la pareja formada por los subtests de Vocabulario y Cubos es la que mejor estima el CIT $\left(r{ }^{\prime}{ }_{s f}=.88\right)$ y el índice de Capacidad General (ICG) $\left(r{ }^{\prime}{ }_{s f}=.93\right)$.

En las Tablas 3 y 4 se resumen las principales formas abreviadas desarrolladas para las distintas versiones de la Escala de Inteligencia de Wechsler para Adultos.

\section{Discusión}

Las sucesivas aparición de versiones de la Escala de inteligencia de Wechsler para adultos ha provocado el desarrollo paralelo de distintas formas abreviadas que permitiesen estimar el CI con un menor tiempo de administración. Los estudios revisados ponen de manifiesto que se utilizan dos métodos de reducción de la escala completa. El primer método consiste en reducir el número de tests dejando cada test intacto. El segundo consiste en reducir el número de ítems de cada test, dejando intacta la estructura de la escala. Según las investigaciones que compararon formas abreviadas basadas en ambos métodos de reducción (Boone, 1992; Robiner, Dossa y O’Dowd, 1988; Watkins, Hilmmel, Pork y Reinberg, 1988), se puede concluir que, aunque ambas formas de proceder presentan unos índices de validez semejantes, las formas abreviadas basadas en la selección de tests superan en fiabilidad a las de reducción de ítems y, además, estiman de manera más ajustada el CI (López et al., 2003).

Sobre las distintas formas abreviadas cabe destacar que alcanzó gran popularidad, para la WAIS, la propuesta de Satz y Mogel (1962), basada en el procedimiento de reducción de ítems. 
Respecto a las formas cortas de la WAIS-R, diversos autores coinciden en afirmar que las formas cortas desarrolladas por Kaufman et al. (1991) constituyen medidas bastante precisas de estimación del CIT. Si bien otros autores destacan la propuesta de Ward (1990) como forma de estimación precisa del CIT en muestras de pacientes psiquiátricos.

Con la aparición de la WAIS-III surgieron nuevas propuestas de formas cortas, como las realizadas por Blyler et al. (2000) para la evaluación de la capacidad intelectual en pacientes diagnosticados de esquizofrenia o la de Christensen et al. (2007) para su uso en población psiquiátrica. Aunque también se continúan realizando estudios basados en la propuesta de Ward (1990) de siete subtests en la WAIS-III, como el de Pilgrim et al. (1999) y el de Axelrod et al. (2001). Los resultados que se extrajeron de estos últimos estudios apoyan el uso de la forma corta de siete subtests de la WAIS-III.

Entre todas las propuestas de formas abreviadas realizadas para esta versión, Girard et al. (2010) concluyen que la versión de Axelrod et al. (2001) es la más adecuada en la estimación del CIV, CIM y CIT teniendo en cuenta la combinación de propiedades psicométricas y disminución del tiempo de administración. Aunque añaden que la de Christensen et al. (2007) representa mejor los cuatro factores de la escala y permite obtener medidas de la velocidad de procesamiento.

Respecto a la última versión, la WAIS-IV, todavía existen pocos estudios sobre formas abreviadas. Meyers et al. (2013) examinaron la utilidad de la forma abreviada propuesta por Ward (1990) para la WAIS-IV en una muestra clínica, concluyendo que es posible utilizarla como una forma corta de la WAIS-IV. Otros autores han propuesto formas cortas compuestas por una pareja de subtests, bien sin restricción previa acerca de los índices que se desea reflejar en la forma corta, basándose únicamente en las mejores combinaciones de parejas de subtests, como en el estudio de Girard et al. (2014) o bien acotando de antemano los índices que debían quedar reflejados en la forma corta resultante, como en el estudio de Denney et al. (2015).

En nuestro país se han desarrollado dos formas cortas de las versiones de la escala. Robles et al. (1990) desarrollaron una forma abreviada de la WAIS con una muestra de pacientes psiquiátricos y Fuentes-Durá et al. (2010) propusieron una forma corta de la WAISIII para su uso con pacientes diagnosticados de esquizofrenia.

En general, las formas abreviadas de las distintas versiones de la Escala de inteligencia de Wechsler para adultos constituyen instrumentos útiles en la estimación de la capacidad intelectual contando con la ventaja de poseer un menor tiempo de administración en comparación con la escala completa, lo cual puede resultar de gran utilidad en determinados grupos clínicos cuyas propias características dificulten la aplicación de la escala completa.

No obstante, la decisión final de utilizar o no determinada forma abreviada para estimar la capacidad intelectual de una persona es una cuestión que recae fundamentalmente sobre el juicio clínico del profesional. Éste deberá considerar qué posee mayor importancia en el proceso evaluativo.

\section{Referencias}

Allen, D. N., Huegel, S. G., Seaton, B. E., Goldstein, G., Gurklis, J. A., y van Kammen, D. P. (1998). Confirmatory factor analysis of the WAIS-R in patients with schizophrenia. Schizophrenia Research, 34, 87-94. 
Axelrod, B. N., Ryan, J. J., y Ward, L. C. (2001). Evaluation of seven-subtest short form the WAIS-III in a referred sample. Archives of Clinical Neuropsychology, 16, 1-8.

Benedict, R. H. B., Schretlen, D., y Bobholtz, J. H. (1992). Concurrent validity of three WAIS$\mathrm{R}$ short forms in psychiatric inpatients. Psychological Assessment, 4, 322-328.

Blyler, C. R., Gold, J. M., Iannone, V. N. y Buchanan, R. W. (2000). Short form of the WAISIII for use with patients with schizophrenia. Schizophrenia Research, 46, 209-215.

Boone, D. E. (1992). Evaluation of Kaufman's short forms of the WAIS-R with psychiatric inpatients. Journal of Clinical Psychology, 48, 239-245.

Britton, P. G., y Savage, R. D. (1966). A short form of the WAIS for use with the aged. British Journal of Psychology, 112, 417-418.

Caplan, B. (1983). Abbreviated WAIS forms for a stroke population. Journal of Clinical Neuropsychology, 5, 239-245.

Christensen, B. K., Girard, T. A., y Bagby, R. M. (2007). Wechsler Adult Intelligence Scale Third Edition short form for Index and IQ Scores in a psychiatric population. Psychological Assessment, 19, 236-240.

Crawford, J. R., Allum., S., y Kinion, J. E. (2008). An index-based short form of the WAIS-III with accompanying analysis of reliability and abnormality of differences. British Journal of Clinical Psychology, 47, 215-237.

Cyr, J. J., y Brooker, B. H. (1984). Use of appropriate formulas for selecting WAIS-R short forms. Journal of Consulting and Clinical Psychology, 52, 903-905.

Denney, D. A., Ringe, W. K., y Lacritz, L. H. (2015). Dyadic short forms of the Wechsler Adult Intelligence Scale-IV. Archives of Clinical Neuropsychology, 30, 404-412.

Doll, E. A. (1917).A brief Binet-Simon scale. Psychology Clinical, 11, 197-211.

Doppelt, J. E. (1956). Estimating the Full Scale score on the Wechsler Adut Intelligence Scale from scores on four subtests. Journal of ConsultingPsychology, 20, 63-66.

Fuentes-Durá, I., Romero-Peris, M., Dasí-Vivó, C., y Ruiz-Ruiz, J. C. (2010). Versión abreviada del WAIS-III para su uso en la evaluación de pacientes con diagnóstico de esquizofrenia. Psicothema, 22, 202-207.

Girard, T. A., Axelrod, B. N, Patel, R., y Crawford, J. R. (2014). Wechsler Adult Intelligence Scale-IV dyads for estimating global intelligence. Assessment, 1-8.

Girard, T. A., Axelrod, B. N. y Wilkins, L. K. (2010). Comparison of WAIS-III Short Forms for Measuring Index and Full-Scale Scores. Assessment, 17, 400-405.

Guertin, W. H., Rabin, A. I., Frank, G. H., y Ladd, C. E. (1961). Research with the Wechsler Intelligence Scales for Adults; 1955-1960. Psychological Bulletin, 59, 1-26.

Kaufman, A. S. (1990). Assessing adolescent and adult intelligence. Boston: Ally \& Bacon.

Kaufman, A. S., Ishikuma, T., y Kaufman-Packer, J. L. (1991). Amazingly short forms of the WAIS-R. Journal of Psychoeducational Assessment, 9, 4-15.

Kaufman, A. S. y Lichtenberger, E. O. (2006). Assessing adolescent and adult intelligence. Hoboken, NJ: Wiley.

López, M. F., Rodríguez, J. M., Santín, C. y Torrico, E. (2003). Utilidad de las formas cortas de la Escala de Inteligencia de Wechsler para Adultos (WAIS). Anales de Psicología, 19, 53-63.

Maxwell, E. (1957) Validities of abbreviated WAIS Scales. Journal of Consulting Psychology, 21, 121-126. 
McCusker, P. J. (1994). Validation of Kaufman, Ishikuma and Kaufman-Parker's Wechsler Adult Intelligence Scale-Revised short forms on a clinical sample. Psychological Assessment, 6, 246-248.

Meyers, J. E., Zellinger, M. M., Kockler, T., Wagner, M., y Mellado-Miller, R. (2013). A validated seven-subtest short form for the WAIS-IV. Applied Neuropsychology: Adult, 20, 249-256.

Missar, C. D., Gold, J. M., y Goldberg, T. E. (1994). WAIS-R short forms in chronic schizophrenia. Schizophrenia Research, 12, 247-250.

Paolo, A. R., y Ryan, J. J. (1991). Application for WAIS-R short to persons 75 years of age and older. Journal of Psychoeducatinal Assessment, 9, 345-352.

Pilgrim, B. M., Meyers, J. E., Bayless, J., y Whetstone, M. M. (1999).Validation of the Ward seven-subtest WAIS-III short form in a neuropsychological population. Applied Neuropsychology, 6, 243-246.

Rabin, A. I. (1943). A short form of the Wechsler-Bellevue test. Journal of Applied Psychology, 27, 320-324.

Reynolds, C. R., Willson, V. L., y Clark, P. L. (1983). A four-subtest short form of the WAISR for clinical screening. The Clinical Neuropsychologist, 5, 111-116.

Robiner, W. D., Dossa, D., y O'Dowd, W. (1988). Abbreviated WAIS-R procedures: use and limitations with head-injured patients. The Clinical Neuropsychologist, 2, 365-374.

Robles, J. I., Ramón, F. J. y Cabrera, J. (1990). Justificación válida para el uso de la fórmula reducida del WAIS propuesta por Britton y Savage (1966). Psiquis, 11, 59-60.

Ryan, J. J., López, S. J., y Werth, T. H. (1998). Administration time estimated for WAIS-III subtest, scales, and short forms in a clinical sample. Journal of Psychoeducational Assessment, 16, 315-323.

Ryan, J. J., Weilage, M. E., y Spaulding, W. D. (1999). Accuracy of the seven subtest WAIS-R short form in chronic schizophrenia. Schizophrenia Research, 39, 79-83.

Satz, P., y Mogel, S. (1962). An Abbreviation of the WAIS for clinical use. Journal of Clinical Psychology, 18, 77-78.

Silverstein, A. B. (1970). Reappraisal of the validity of WAIS, WISC and WPPSI short forms. Journal of Consulting and Clinical Psychology, 34, 12-14.

Silverstein, A. B. (1982). Two and four subtests short forms of the Wechsler Adult Intelligence Scale-Revised. Journal of Consulting and Clinical Psychology, 50, 415-418.

Sumiyoshi, C., Uetsuki, M., Suga, M., Kasai, K., y Sumiyoshi, T. (2013). Development of brief versions of the Wechsler Intelligence Scale for schizophrenia: considerations of the structure and predictability of intelligence. Psychiatry Research, 210, 773-779.

Terman, L. M., y Merrill, M. A. (1937). Measuring intelligence. Boston: Houghton Mifflin.

Ward, L. C. (1990). Prediction of verbal, performance, and full scale IQs from seven subtests of WAIS-R. Journal of Clinical Psychology, 46, 436-440.

Ward, L. C., Selby, R. B., y Clark, B. L. (1987). Subtest administration times and short forms of the WAIS-R. Journal of Clinical Psychology, 43, 276-278.

Watkins, C. E., Hilmmel, C. D., Pork, N. E., y Reinberg, J. A. (1988). WAIS-R short forms with mentally retarded adults: a note of caution. Journal of Mental Deficiency Research, 32, 239-234. 
Wechsler, D. (1939). Wechsler-Bellevue intelligence scale. New York: Psychological Corporation.

Wechsler, D. (1955). Wechsler adult intelligence scale. New York: Psychological Corporation. Wechsler, D. (1981). Wechsler adult intelligence scale-revised. San Antonio, TX: Psychological Corporation.

Wechsler, D. (1997). Wechsler adult intelligence scale-III. San Antonio, TX: Psychological Corporation

Wechsler, D. (2008). Escala de Inteligencia de Wechsler para Adultos - Cuarta Edición: Manual técnico y de interpretación. Madrid: Pearson.

Wolfson, W., y Bachelis, L. (1960). An abbreviated form of the WAIS verbal scale. Journal of Clinical Psychology, 16, 421.

Zimmerman, I. L., y Woo-Sam, J. M. (1973). Clinical interpretation of the Wechsler Adult Intelligence Scale. New York: Grune \& Stratton. 